\title{
Research Article \\ Effect of Discrete Levels Width Error on the Optical Performance of the Diffractive Binary Lens
}

\author{
Manal Alshami, Mohamed Fawaz Mousselly, and Anas Wabby \\ Higher Institute for Applied Sciences and Technology, Damascus, Syria \\ Correspondence should be addressed to Manal Alshami; loly_394@hotmail.com
}

Received 8 December 2016; Accepted 27 December 2016; Published 17 January 2017

Academic Editor: Chenggen Quan

Copyright ( 2017 Manal Alshami et al. This is an open access article distributed under the Creative Commons Attribution License, which permits unrestricted use, distribution, and reproduction in any medium, provided the original work is properly cited.

\begin{abstract}
The effects of discrete levels width error developed by thin film deposition on the optical performance of diffractive binary germanium lens with four discrete levels are investigated using nonsequential mode in the optical design code ZEMAX. The thin film deposition technique errors considered are metallic mask fabrication errors. The peak value of the Point Spread Function (PSF) was used as criterion to show the effect of discrete levels width error on the optical performance of the four-level binary germanium lens.
\end{abstract}

\section{Introduction}

To enhance optical resolution and reduce aberrations, refractive and diffractive lenses are often combined as a hybrid lens [1]. Diffractive lenses are essentially gratings with variable groove spacing across the optical surface, which impart a change in phase of the wavefront passing through it [2].

A diffractive optical element (DOE), with continuous surface profile, is often referred to as kinoform, and the theoretical ideal profile of the diffractive surface can be approximated in a discrete fashion (the sag is approximated by discrete steps), similar to the digital representation of an analog function [3]. This discrete representation is called a multilevel or binary profile [4]. The design techniques used for binary optics were initially developed by integrated circuit (IC) manufacturers, by using the CAD software [4].

Diffractive surfaces in most of the optical design codes, such as Oslo [5] and ZEMAX [2], are closer approximation to kinoforms than true binary optics, since the phase is continuous everywhere, so the evaluation of the optical performance of that elements will be done for continuous phase profile case [6].

Swanson [3] has developed a technique using Optical Research Associates' (ORA's) Code V lens design software [7] to design diffractive optical elements. This is possible because the code uses direct ray trace with a subcode for holographic optical elements (HOEs), which can be used to partially simulate binary elements. The finite-difference time-domain method was used to simulate subwavelength diffractive lenses $[8,9]$. But, for a few of the optical designers who use the optical design code ZEMAX, it will be preferable to design diffractive binary lens by it, but the optical design code ZEMAX does not model the wavelength-scale grooves directly. Instead, ZEMAX uses the phase advance or delay represented by the surface locally to change the direction of propagation of the ray [2].

The fabrication of the single-level and multilevel diffractive lenses involves the generation of a set of masks that are used sequentially for the transfer of their pattern to a substrate in conjunction with photoresist deposition, exposure, and development, as well as an etching procedure such as reactive-ion etching [10], or with thin film deposition [11, 12].

For example, $K$ masks are needed for a lens of $2^{K}$ phase levels [3]. The development of these masks (photoresist [11] or metallic [12]) is not usually error-free. These errors are usually known as mask fabrication errors and cause significant deformations of the resulting diffractive binary lens surface and corresponding deterioration of the lens performance. Therefore, the analysis of the effects of these errors on the performance of diffractive optical elements and the determination of acceptable fabrication tolerances for each design is of central importance.

Choi and others had used geometrical and Fourier optics theory to simulate the decrease of MTF due to diffractive 
TABLE 1: Specifications of the refractive lens ( $\mathrm{mm}$ ).

\begin{tabular}{lccccc}
\hline Surface & Type & Radius & Thickness & Glass & Diameter \\
\hline OBJ & Standard & Infinity & Infinity & & 0.000 \\
STO & Standard & 225.371 & 5.000 & Germanium & 33.097 \\
2 & Standard & Infinity & 72.849 & & 32.787 \\
IMA & Standard & Infinity & & & 13.435 \\
\hline
\end{tabular}

optical element fabrication error [1]; Glytsis and others [10] had used the Boundary-Element Method (BEM) as basic modeling tool for analyzing diffractive lenses with fabrication errors. The effects of fabrication errors on the predicted performance of surface-relief phase gratings are analyzed with a rigorous vector diffraction technique by Pommet et al. [13]. Jabbour had used the method of generalized projection to study the effect of experimental errors on the diffractive optical element performance [14].

Alshami et al. [12] had used metallic masks in the development of binary diffractive germanium lens by thin film deposition; hopefully, this paper shows the effect of discrete levels width error due to metallic mask fabrication errors on the optical performance using nonsequential mode in ZEMAX to design the four-level binary surface of a diffractive germanium lens, where the first part presents the design of 4-step binary surface of a diffractive germanium lens [12] with nonsequential mode in ZEMAX and the second part presents the effect of discrete levels width error due to the mask fabrication on the optical performance using the peak value of PSF as criterion.

\section{Design of Four-Step Binary Surface in ZEMAX}

The design of four-step binary surface of the diffractive binary germanium lens [12] by nonsequential mode in ZEMAX will be presented via the following procedure.

2.1. Refractive Lens. Table 1 shows the optical design specifications of refractive germanium (planoconvex) lens, as shown in Figure 1, for the wavelength band (8)-(12) $\mu \mathrm{m}$, effective focal length of $75 \mathrm{~mm}$ with a 9.09-degree field of view, and diameter $33 \mathrm{~mm}$.

2.2. Diffractive Lens. Table 2 shows the optical design specifications of diffractive germanium lens, with the same conditions as refractive one, and the plane surface chosen as the binary 2 surface (1), as shown in Figure 2:

$$
\emptyset=-0.65554 \rho^{2}+8.97589 \rho^{4} .
$$

2.3. Switch from Kinoform to Binary Surface. In the optical design of the considered lens [12], the diffractive surface contained one diffractive zone, and the ideal diffractive phase profile to be approximated in a binary fashion (4 steps or 4 phase levels) is (1). The diameter of each discrete phase level or binary step (equivalent to phase value $\pi / 2, \pi, 3 \pi / 2,2 \pi$ ) and the sag's thickness equivalent to each phase value are shown in Table 3 and Figure 3 [12].

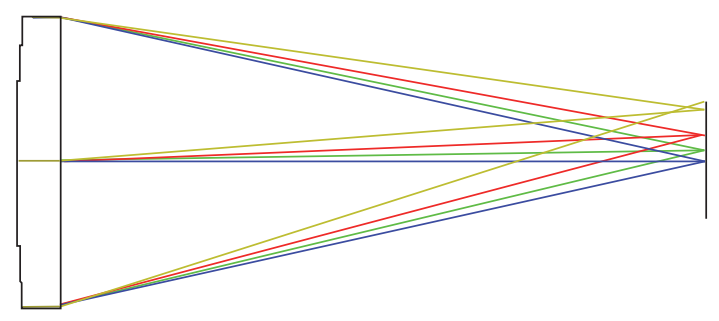

FIGURE 1: Layout of the refractive lens.

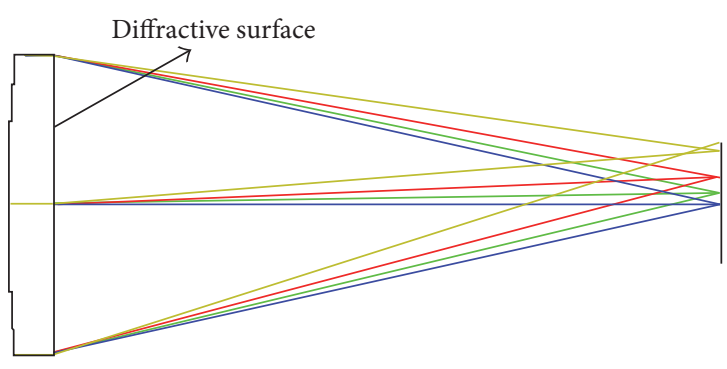

FIGURE 2: Layout of the diffractive lens.

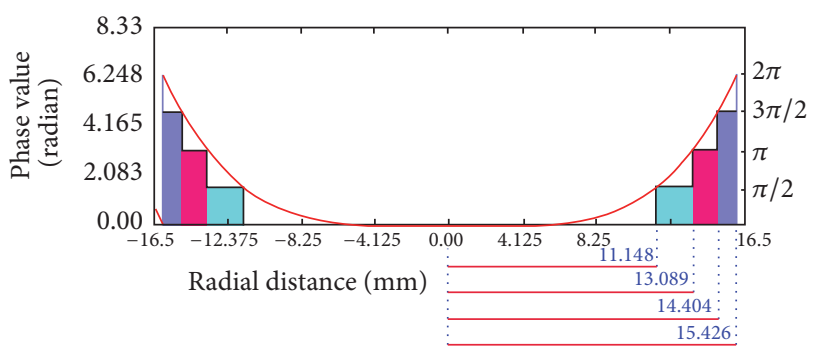

FIGURE 3: Phase curve versus aperture of the diffractive surface slicing into $2 \pi$ layers and the discrete phase levels.

2.4. Design of Four-Step Binary Surface of a Diffractive Germanium Lens. The design of four-step binary surface of diffractive germanium lens by nonsequential mode in ZEMAX is presented in Tables 4 and 5, and it was done by using the object cylinder volume which is a rotationally symmetric volume to design each step of germanium, where the diameter of the front and rear face of each cylinder is the same as the equivalent binary step and the length along the local $z$-axis of each cylinder is the thickness of the equivalent binary step, as shown in Figure 4. For the optical design in nonsequential mode, we need to define $x, y, z$ position of each object.

Figure 5 shows the difference in FFT PSF cross-sectional curves between the refractive, diffractive, and the designed four-step binary germanium lens.

\section{Effect of Discrete Levels Width Error on the Diffractive Optical Element Performance}

Imprecision in the metallic mask fabrication process can cause the width of the discrete levels to differ from the theoretical target. This can have an adverse effect on the optical performance. To understand how this effect degrades 
TABLE 2: Specifications of the diffractive lens $(\mathrm{mm})$.

\begin{tabular}{|c|c|c|c|c|c|c|c|}
\hline Surface & Type & Radius & Thickness & Glass & Diameter & Coeff. on $\rho^{2}$ & Coeff. on $\rho^{4}$ \\
\hline OBJ & Standard & Infinity & Infinity & & 0.000 & & \\
\hline STO & Standard & 225.371 & 5.000 & Germanium & 33.097 & & \\
\hline 2 & Binary 2 & Infinity & 73.339 & & 32.787 & -0.65554 & 8.97588 \\
\hline IMA & Standard & Infinity & & & 13.323 & & \\
\hline
\end{tabular}

TABLE 3: Diameters and thickness of each binary zone.

\begin{tabular}{lcccc}
\hline $\begin{array}{l}\text { Binary zone's } \\
\text { number }\end{array}$ & $\begin{array}{c}\text { Equivalent phase value } \\
(\text { radian})\end{array}$ & $\begin{array}{c}\text { Radius of each binary zone } \\
(\mathrm{mm})\end{array}$ & $\begin{array}{c}\text { Diameter of each binary zone } \\
(\mathrm{mm})\end{array}$ & $\begin{array}{c}\text { Equivalent sag's thickness } \\
(\mu \mathrm{m})\end{array}$ \\
\hline 1 & $\pi / 2$ & 11.148 & 22.295 & 0.833 \\
2 & $\pi$ & 13.089 & 26.177 & 1.667 \\
3 & $3 \pi / 2$ & 14.404 & 28.807 & 2.498 \\
4 & $2 \pi$ & 15.426 & 30.851 & 3.333 \\
\hline
\end{tabular}

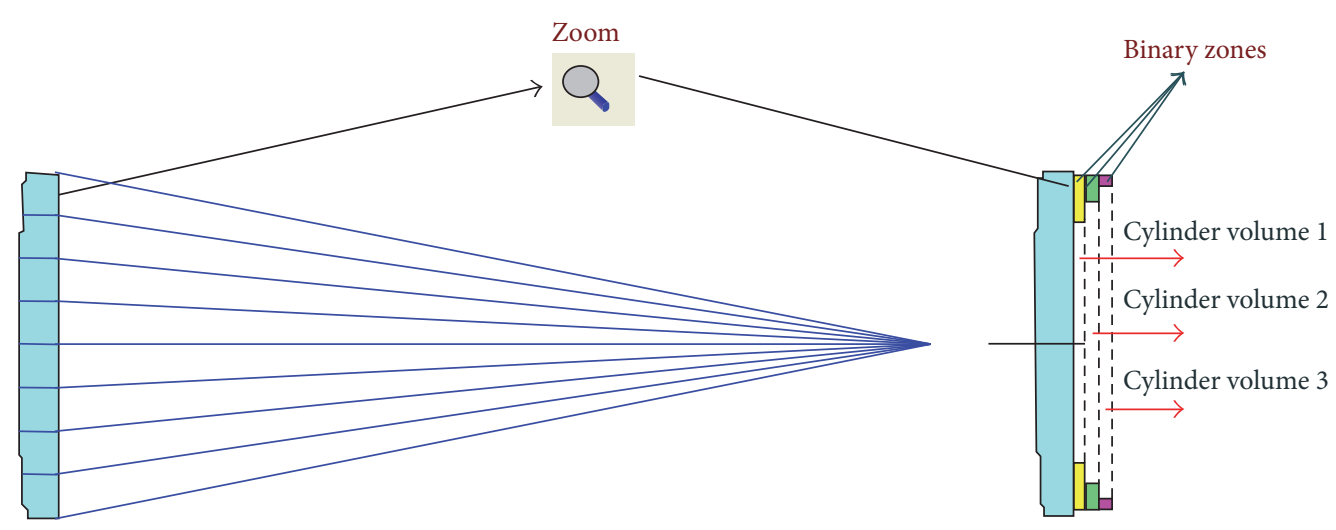

FIGURE 4: The binary diffractive lens with discrete phase levels.

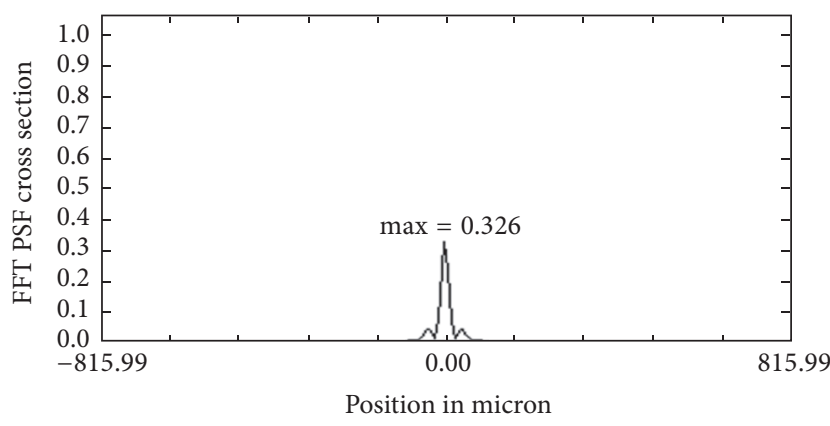

(a)

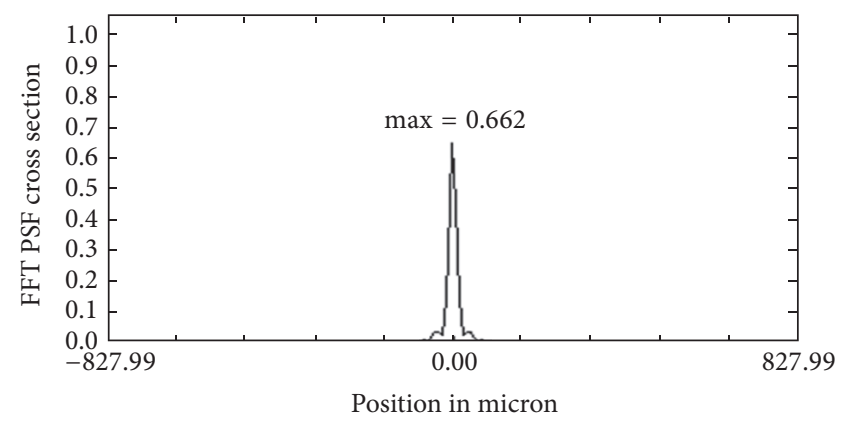

(b)

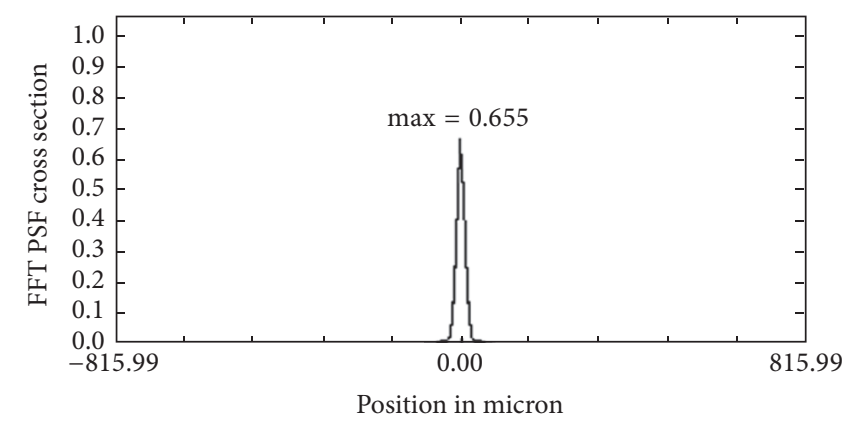

(c)

FIGURE 5: FFT PSF cross section of (a) refractive lens, (b) diffractive lens, and (c) designed binary lens. 
TABLE 4: Optical design of the binary germanium lens in nonsequential mode.

\begin{tabular}{lccccc}
\hline Surface & Type & Radius & Thickness & Glass & Diameter \\
\hline OBJ & Standard & Infinity & Infinity & & Infinity \\
STO & Standard & 225.371 & 5.000 & Germanium & 33.097 \\
2 & Standard & Infinity & 0.000 & & 32.787 \\
3 & Nonsequential & Infinity & & 32.787 & 73.368 \\
IMA & Standard & Infinity & & & 13.342 \\
\hline
\end{tabular}

TABLE 5: Data in the nonsequential component editor.

\begin{tabular}{|c|c|c|c|c|c|c|}
\hline Object number & Object 1 & Object 2 & Object 3 & Object 4 & Object 5 & Object 6 \\
\hline Object type & $\begin{array}{c}\text { Standard } \\
\text { lens }\end{array}$ & $\begin{array}{l}\text { Cylinder } \\
\text { volume }\end{array}$ & $\begin{array}{c}\text { Standard } \\
\text { lens }\end{array}$ & $\begin{array}{l}\text { Cylinder } \\
\text { volume }\end{array}$ & $\begin{array}{c}\text { Standard } \\
\text { lens }\end{array}$ & $\begin{array}{l}\text { Cylinder } \\
\text { volume }\end{array}$ \\
\hline$Z$ position $(\mathrm{mm})$ & 0.000 & 0.000 & 0.000833 & 0.000833 & 0.001667 & 0.001667 \\
\hline Material & Germanium & & Germanium & & Germanium & \\
\hline Front $R(\mathrm{~mm})$ & 0.000 & 11.148 & 0.000 & 13.089 & 0.000 & 14.404 \\
\hline$Z$ length (mm) & 0.000 & 0.000833 & 0.000 & 0.000833 & 0.000 & 0.000833 \\
\hline $\operatorname{Back} R(\mathrm{~mm})$ & 16.500 & 11.148 & 16.500 & 13.089 & 16.500 & 14.404 \\
\hline Edge $1(\mathrm{~mm})$ & 16.500 & Not used & 16.500 & not used & 16.500 & Not used \\
\hline Thickness (mm) & 0.000833 & Not used & 0.000833 & not used & 0.000833 & Not used \\
\hline Clear $2(\mathrm{~mm})$ & 16.500 & Not used & 16.500 & not used & 16.500 & Not used \\
\hline Edge $2(\mathrm{~mm})$ & 16.500 & Not used & 16.500 & not used & 16.500 & Not used \\
\hline
\end{tabular}

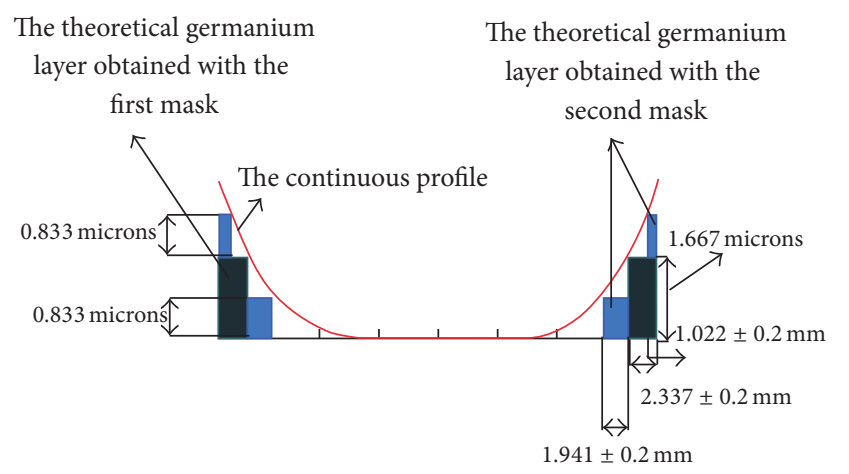

FIGURE 6: Germanium layers (binary zones) and the expected error in their width.

performance and thus obtain a tolerance for fabrication errors, we studied how the peak value of the PSF of the designed lens changes as a function of discrete level or zone width variation. The variable $\Delta w$ is introduced to specify the difference between the final and intended position of the boundaries of each binary zone (an expected error in the width of each binary zone will result due to the metallic masks fabrication accuracy $0.1 \mathrm{~mm}$ of the laser machine), as in Figure 6 [12]; the width of each binary zone is then changed by $2 \Delta w(2 \Delta w=0.2 \mathrm{~mm})$. The sign of $\Delta w$ can be positive or negative corresponding to wider and narrower zones, respectively. In this study, it is assumed that $\Delta w$ is equal for all zones, independent of their width.

\section{Results and Discussion}

Table 6 and Figure 7 show the variation in peak value of PSF as a function of $2 \Delta w$. The change in $2 \Delta w$ studied was limited to

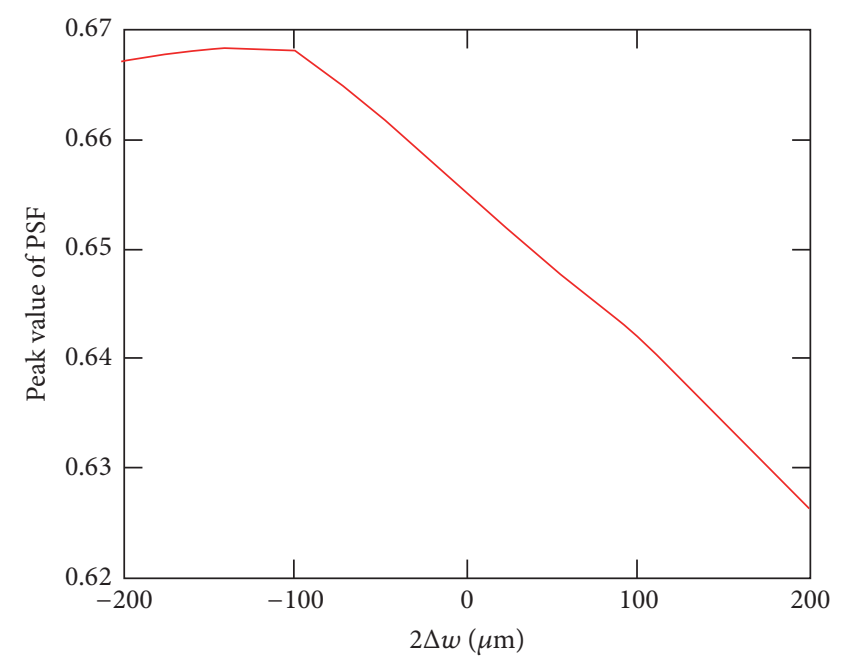

FIGURE 7: Peak value of PSF as a function of variation in zone width error.

$200 \mu \mathrm{m}(\Delta w=100 \mu \mathrm{m}$, the metallic masks fabrication error caused by laser machine). The change $200 \mu \mathrm{m}$ in $2 \Delta w$ has the effect of lowering the PSF peak value (Table 6) by $5 \%$, lowering the diffraction efficiency by $5 \%$ [15].

Figure 8 shows the FFT PSF cross section of the considered lens for the extreme error values and without error. It can be seen from Figure 8 that, for this particular diffractive binary lens, the axial resolution increases with increasing zone width, but this occurs at the expense of decreasing the PSF peak value. The metallic mask can be replaced with similar dimension masks which can be produced by threedimensional printer (rapid prototype) with accuracy of $35 \mu \mathrm{m}$ so the discrete levels width error will change from $-70 \mu \mathrm{m}$ to 
TABLE 6: Peak value of PSF as a function of $2 \Delta w$.

\begin{tabular}{|c|c|c|c|c|c|c|c|c|c|}
\hline $2 \Delta w(\mu \mathrm{m})$ & -200 & -150 & -100 & -50 & 0.00 & 50 & 100 & 150 & 200 \\
\hline Peak value of PSF & 0.667 & 0.6684 & 0.6681 & 0.662 & 0.655 & 0.648 & 0.642 & 0.634 & 0.626 \\
\hline
\end{tabular}

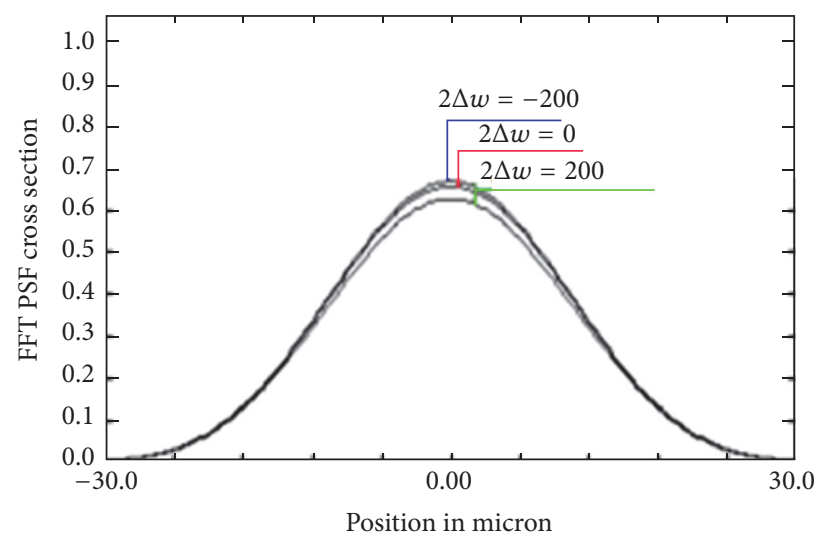

FIGURE 8: FFT PSF cross section.

$70 \mu \mathrm{m}$ which cause lowering in PSF peak value less than $2 \%$ then lowering in diffraction efficiency less than $2 \%$; in this case within the $70 \mu \mathrm{m}$ change in $2 \Delta w$, the performance of the considered lens is still acceptable.

\section{Conclusion}

The effects of discrete levels width error developed by thin film deposition on the optical performance of diffractive binary germanium lens with four discrete levels have been analyzed using nonsequential mode in the optical design code ZEMAX. The thin film deposition technique errors considered are metallic mask fabrication errors. It was found by using the peak value of PSF as criterion that the metallic mask fabrication errors (100 $\mu \mathrm{m}$ laser machine accuracy) have a significant effect on the designed four-level binary germanium lens performance, and to reduce this effect it will be preferable to use another technique to fabricate the desired mask with more fabrication accuracy like, for example, by three-dimensional printer ( $35 \mu \mathrm{m}$ is its accuracy).

\section{Competing Interests}

The authors declare that they have no competing interests.

\section{Acknowledgments}

This work was totally supported by HIAST (Higher Institute for Applied Sciences and Technology).

\section{References}

[1] H. Choi, W.-C. Kim, S.-H. Lee, N.-C. Park, and U. Y.-P. Park, "Effects of fabrication errors in the diffractive optical element on the modulation transfer function of a hybrid lens," Journal of the Optical Society of America A: Optics and Image Science, and Vision, vol. 25, no. 11, pp. 2764-2766, 2008.

[2] Zemax Product, http://www.zemax.com.
[3] G. J. Swanson, Binary Optics Technology, Massachusetts Institute of Technology, Cambridge, Mass, USA, 1989.

[4] A. D. Kathman and S. K. Pitalo, "Binary optics in lens design," in International Lens Design Conference, vol. 1354 of Proceedings of SPIE, 1990.

[5] Lambda Research Corporation, OSLO, version 6.2, 2001.

[6] N.-H. Kim and R. Zemax, "How Diffractive Surfaces are Modeled in Zemax," September 2005.

[7] Code V reference manual, CODE V version 7.10, Optical Research Associates, March 1987.

[8] T. Shirakawa, K. L. Ishikawa, S. Suzuki, Y. Yamada, and H. Takahashi, "Design of binary diffractive microlenses with subwavelength structures using the genetic algorithm," Optics Express, vol. 18, no. 8, pp. 8388-8391, 2010.

[9] V. Raulot, B. Serio, P. Gérard, P. Twardowski, and P. Meyrueis, "Modeling of a diffractive micro-lens by an effective medium method," in Micro-Optics 2010, 77162J, vol. 7716 of Proceedings of SPIE, May 2010.

[10] E. N. Glytsis, M. E. Harrigan, T. K. Gaylord, and K. Hirayama, "Effects of fabrication errors on the performance of cylindrical diffractive lenses: rigorous boundary-element method and scalar approximation," Applied Optics, vol. 37, no. 28, pp. 65916602,1998

[11] J. Jahns and S. J. Walker, "Two-dimensional array of diffractive microlenses fabricated by thin film deposition," Applied Optics, vol. 29, no. 7, pp. 931-936, 1990.

[12] M. Alshami, A. Wabby, and M. F. Mousselly, "Design and development of binary diffractive Germanium lens by thin film deposition," Journal of the European Optical Society, vol. 10, Article ID 15055, 2015.

[13] D. A. Pommet, E. B. Grann, and M. G. Moharam, "Effects of process errors on the diffraction characteristics of binary dielectric gratings," Applied Optics, vol. 34, no. 14, pp. 2430-2435, 1995.

[14] T. G. Jabbour, Design, Analysis, and Optimization of Diffractive Optical Elements under High Numerical Aperture Focusing, University of Central Florida, 2009.

[15] G. J. Swanson and W. B. Veldkamp, "Diffractive optical elements for use in infrared systems," Optical Engineering, vol. 28, no. 6, pp. 605-608, 1989. 

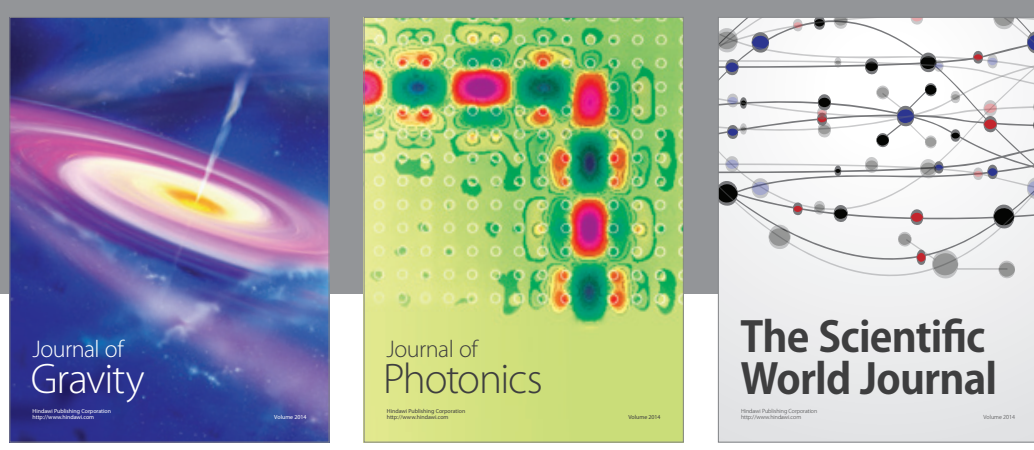

The Scientific World Journal
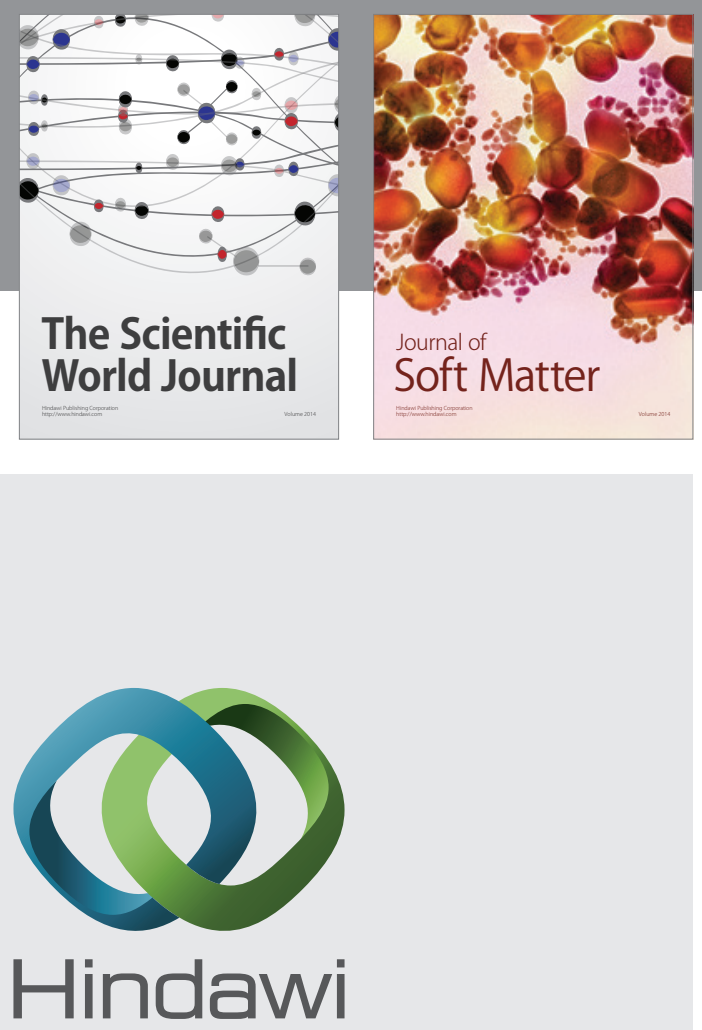

Submit your manuscripts at

https://www.hindawi.com
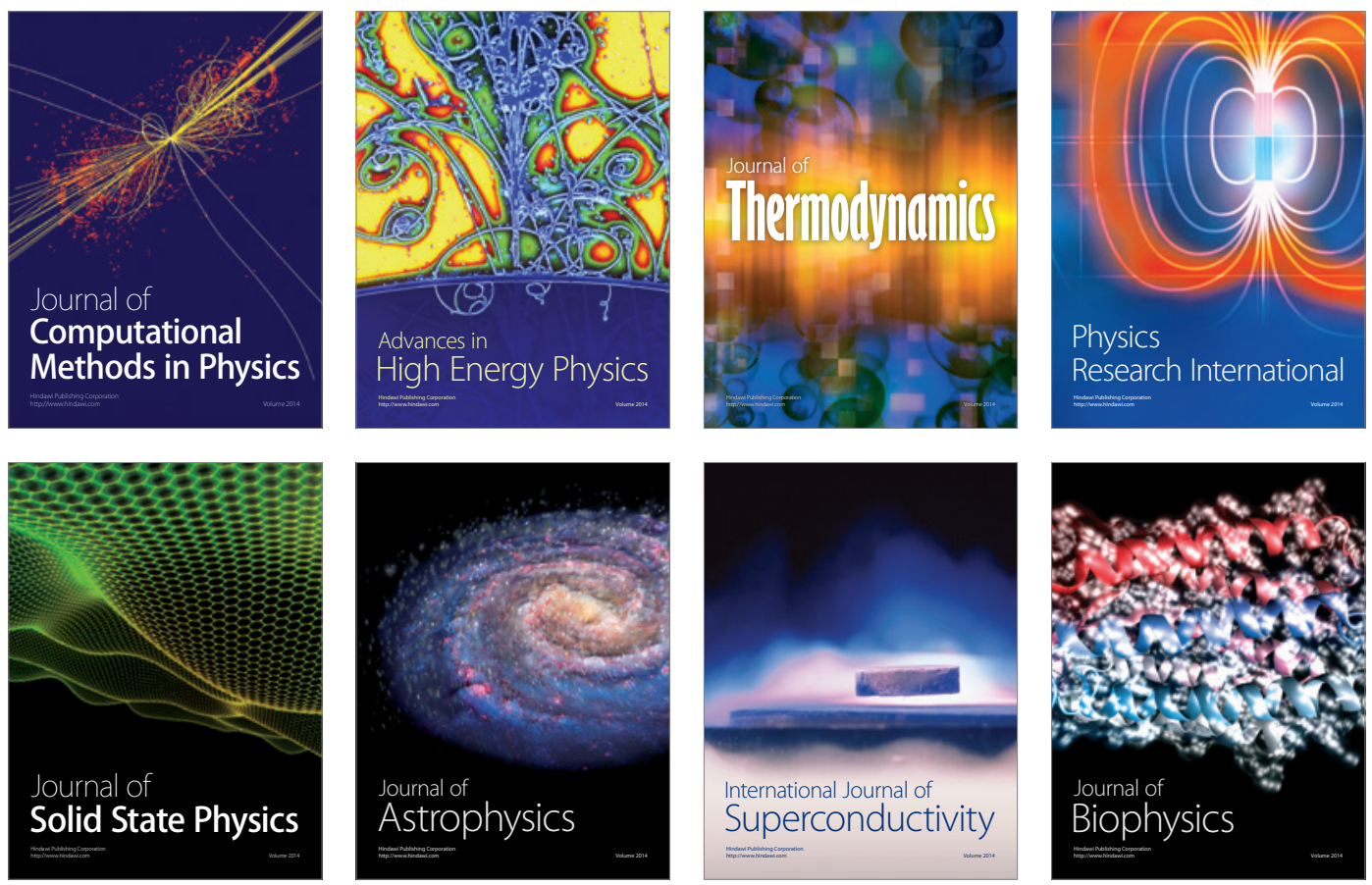
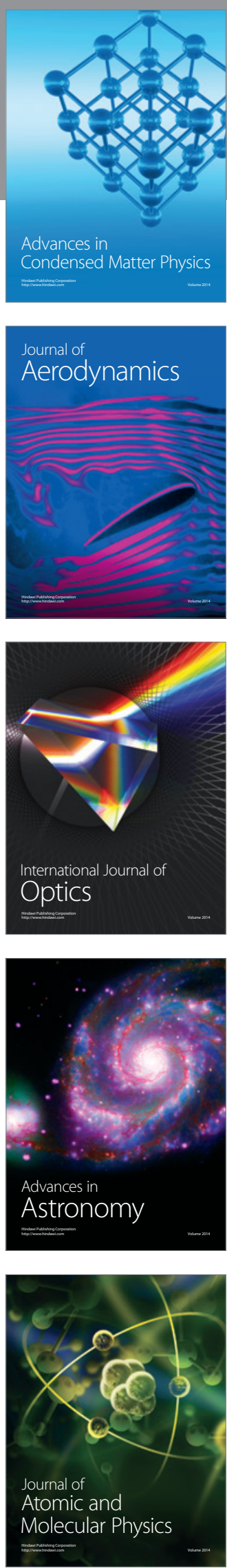